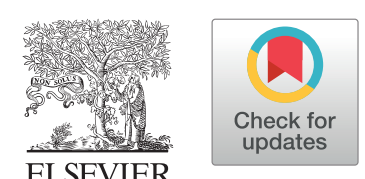

\title{
Review
}

\section{Social Media as a Tool to Advance Women in Cardiology: Paving the Way for Gender Equality and Diversity}

Nooshin Beygui, ${ }^{a}$ Disha Bahl, ${ }^{b, \ddagger}$ Christina Mansour, MD, ${ }^{c, \ddagger}$ Erin D. Michos, MD, MHS, ${ }^{d}$ Poonam Velagapudi, MD, MS, ${ }^{\mathrm{e}}$ Julia Grapsa, MD, PhD, ${ }^{\mathrm{f}}$ Andrew Choi, MD, ${ }^{\mathrm{g}}$

Srihari S. Naidu, MD, ${ }^{\text {h }}$ and Purvi Parwani, MBBS, $\mathrm{MPH}^{\mathrm{i}}$

${ }^{a}$ Central Michigan University College of Medicine, Mount Pleasant, Michigan, USA

${ }^{b}$ St. George's University School of Medicine, Grenada, West Indies

'School of Medicine, Loma Linda University, Loma Linda, California, USA

${ }^{d}$ Division of Cardiology, Johns Hopkins University School of Medicine, Baltimore, Maryland, USA

e University of Nebraska Medical Center, Omaha, Nebraska, USA

${ }^{f}$ Department of Cardiology, Guys and St. Thomas NHS Hospitals Trust, London, United Kingdom

${ }^{g}$ The George Washington University School of Medicine, Washington, DC, USA

${ }^{h}$ Division of Cardiology, Westchester Medical Center, Westchester, New York, USA

${ }^{i}$ Division of Cardiology, Department of Medicine, Loma Linda University Health, Loma Linda, California, USA

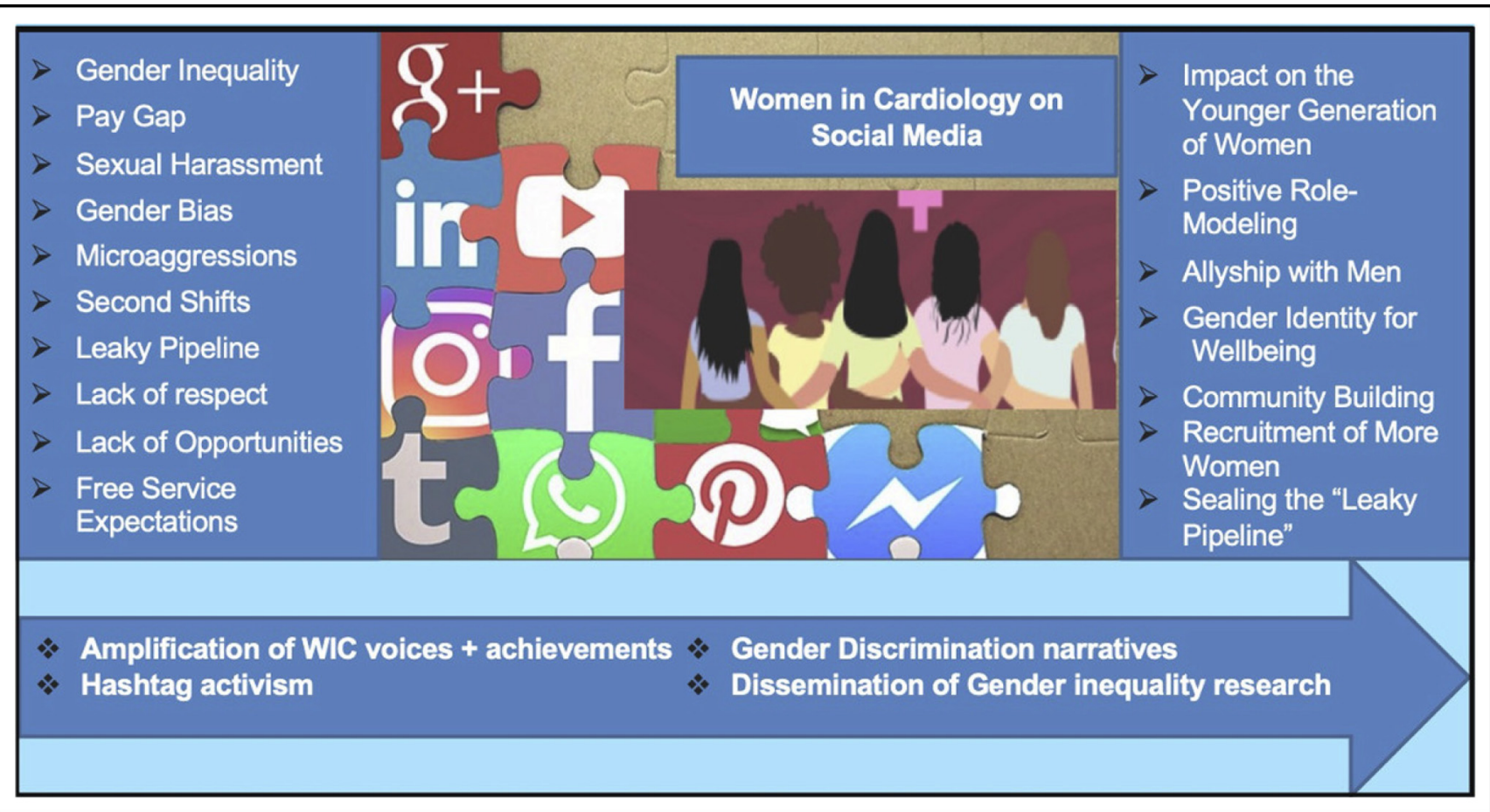

Received for publication June 21, 2021. Accepted August 22, 2021.

Ethics Statement: The research reported has adhered to the relevant ethical guidelines.

¥These authors contributed equally to this work.
Corresponding author: Dr Purvi Parwani, Loma Linda University Medical Centre, 11234 Anderson Street, Loma Linda, California, 92354 USA. Tel.: +1-909-558-9730; fax: +1-909-558-0903.

E-mail: pparwani@llu.edu

See page S135 for disclosure information. 


\begin{abstract}
Although the number of women in the field of medicine continues to rise, the discrimination against women and the gender inequity in both leadership roles and salary remains persistent. The gender divide is particularly prominent in male-dominated specialties, such as cardiology. Social media help foster global connections and disseminate information quickly and worldwide. The rise of social media has influenced how female physicians communicate and has shown its benefits particularly within the field of cardiology. Virtual platforms are important avenues where female physicians have united for greater representation of gender issues and advocacy efforts. Social media further amplify gender-equality activism by facilitating the conversations surrounding gender equity and proposing solutions to self-identified issues by the virtual community of female physicians and their allies. In this review, we discuss the role of social media as tools for advancing women in the field of cardiology and fostering gender equality and diversity.
\end{abstract}

In the past decade, there has been a steady rise in the percentage of women in the field of medicine. According to Association of American Medical Colleges (AAMC) data, the percentage of self-identified women in medical school was $53.6 \%$ in 2020 , up from $46.9 \%$ in 2015 , tipping the scale in favor of female predominance in medical education programs. ${ }^{1}$ Despite this increase, a significant number of women in the medical field still face sex- and gender-based harassment (sex is a biological construct that defines an individual as being a man or a woman according to anatomy, physiology, and genetics; gender is a psychosocial construct and has a wider scope incorporating the effects of social norms and expectations, roles, behaviours, expressions, and identities of women, girls, men, boys, and gender-diverse people in each society ${ }^{2}$ ), microaggressions, and workplace inequality, ${ }^{3}$ suggesting that the rapid rise in matriculation has not translated into the culture change required to improve retention of women in the field of medicine. The National Academies of Sciences, Engineering, and Medicine reported widespread gender-based inequality in classrooms, laboratories, academic medical centers, field sites, observatories, and conferences. ${ }^{4}$ Women face discrimination and are met with social and institutional barriers that can negatively impact their work and mental health, ultimately resulting in skilled, educated, professional women leaving science careers. ${ }^{5}$ Among the fields most affected, medicine ranked at the top in terms of sexual harassment and discrimination. ${ }^{6}$ In a recent investigation of the components and impact of a hostile work environment among cardiologists, it was determined that $56 \%$ experienced discrimination, $43 \%$ experienced emotional harassment, and $12 \%$ experienced sexual harassment. These events negatively impacted patient care and interaction with colleagues. ${ }^{6}$ In this report, the "high-risk sexual harassment workplaces" within the STEMM fields (science, technology, engineering, math, and medicine), such as cardiology, were found to be directly

\section{RÉSUMÉ}

Bien que le nombre de femmes dans le domaine de la médecine continue d'augmenter, la discrimination à l'égard des femmes et l'inégalité entre les sexes, tant au niveau des rôles de direction que des salaires, persistent. L'écart entre les sexes est particulièrement marqué dans les spécialités à prédominance masculine, comme la cardiologie. Les médias sociaux contribuent à favoriser les liens à l'échelle mondiale et permettent de diffuser des renseignements rapidement dans le monde entier. L'essor des médias sociaux a influencé la manière dont les femmes médecins communiquent, et ces médias ont démontré des bienfaits, notamment dans le domaine de la cardiologie. Les plateformes virtuelles sont des moyens importants utilisés par les femmes médecins pour s'unir afin de mieux mettre de l'avant les questions de genre et d'améliorer les efforts de sensibilisation. Les médias sociaux amplifient encore davantage le militantisme en faveur de l'égalité des sexes en facilitant les conversations autour de l'égalité des sexes et en proposant des solutions aux problèmes ciblés par la communauté virtuelle des femmes médecins et de leurs alliés. Dans cet examen, nous discutons du rôle des médias sociaux à titre d'outils permettant de faire progresser les femmes dans le domaine de la cardiologie et de favoriser l'égalité des sexes et la diversité.

correlated to "male domination and organizational tolerance," in comparison to gender-balanced workgroups. This perhaps suggests that the medical field, and some specialties more than others, perpetuates an environment that in totality is damaging to women's careers. ${ }^{4}$

The percentage of women in some medical specialties, such as cardiology and orthopedic surgery, is low compared to that of their male counterparts. Although women comprise $>$ $50 \%$ of medical school trainees, there is a notable decline in women in cardiovascular medicine as they progress beyond medical school, with only $43 \%$ of internal medicine residents, $25.2 \%$ of cardiovascular disease fellows, and $13 \%$ of interventional cardiology fellows in 2019 being women. ${ }^{7}$ From 2019-2020, the Accreditation Council for Graduate Medical Education (ACGME) reported that general cardiology fellowship training was ranked as being one of the worst in terms of underrepresentation of women among medical subspecialities. ${ }^{8}$ Similarly, there are fewer women cardiologists, as they comprise $15 \%$ of active physicians within cardiovascular disease, and $8 \%$ within interventional cardiology. Of 3810 cardiologists with faculty appointments, only 630 were noted to be women in 2014. ' Women are also underrepresented in various leadership roles. Between 1998 and 2018, there were no female editors-in-chief for US general cardiology journals, and only 1 for general European cardiology journals. ${ }^{10}$ Although there has been an increase in women serving on the editorial boards for Circulation, Journal of the American College of Cardiology, and American Journal of Cardiology taken together, the overall level of representation remains low, with only 2 editors-in-chief being women in over 20 years, with 2 more women recently named as such for 2019, and a coauthor (E.D.M.) of this piece becoming editorin-chief in 2021 at the American Journal of Preventive Cardiology. ${ }^{10}$ Lack of female representation in leadership positions within cardiology also creates a dearth of female mentors 


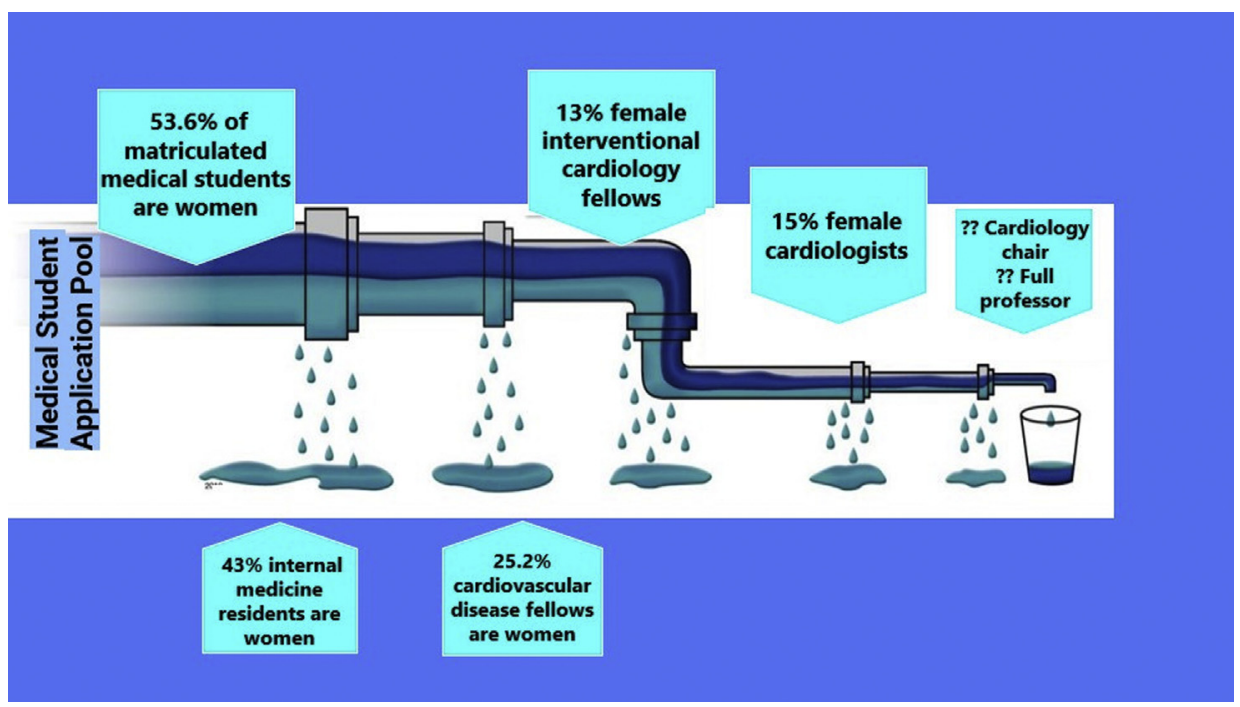

Figure 1. "The Leaky Pipeline" ${ }^{1,4,6,7}$ : the diagram outlines the gradual decline in the number of women-from the majority in medical school to a minority within cardiology subspecialties, and with only a small proportion in academic leadership positions. WIC, women in cardiology.

for trainees, a significant power differential, and translates into fewer opportunities for women to be heard and make an impact. This effect is known as the "leaky pipeline," as women do not follow the hierarchical path or "pipeline" of moving consistently toward senior positions ${ }^{5}$ (Fig. 1). In addition to the leadership disparities, women continue to be paid less than their male colleagues, despite spending more time with their patients. Although the degree of wage discrepancy varies among subspecialties, significant sex differences in salary have been demonstrated within the majority of subspecialties, and this difference persists even after statistical adjustments for age, experience, specialty, faculty rank, and measures of research productivity and clinical revenue. ${ }^{11}$ The gap is particularly prominent in cardiology, a field in which, over a 35-year career, a woman cardiologist in the US is estimated to earn $\$ 2.5$ million less than a male cardiologist. ${ }^{12}$ Notably, a 2016 study reported that among all other subspecialties, after adjusting for years of experience and hours worked, cardiology ranked top among fields with the largest absolute sex-based differences in physician salary. The astounding point to be taken from these studies remains the fact that for equal work, there is a significant lack of equal pay, despite adjusting for all other potential external variables, such as age, academic degree, specialty, and number of sessions per week or day. ${ }^{1}$

Gender-based disparities in professional lives are compounded by those in personal lives, an effect termed "the second shift" by sociologist Arlie Hochschild. Women in cardiology (WIC) more often face the challenges of disparities in not only their professional life, but also their personal life, particularly in relation to household duties such as cleaning, caring for children, and cooking. ${ }^{13,14}$ This imbalance creates a further disadvantage for women who not only are trying to excel professionally, but also feel the additional pressure of taking care of domestic duties at home. ${ }^{15}$ Physician mothers in both procedural and nonprocedural specialties reported having primary responsibility for most domestic tasks in a survey of 1712 attending physician mothers. ${ }^{16}$ This study also found that increased responsibility at home was associated with career dissatisfaction or a desire to change careers. ${ }^{13,14,17}$

Gender inequalities in specialties within the field of medicine are extensive. ${ }^{15,18}$ There is growing evidence that these issues and the inequality described above create a complex interplay of institutional and societal biases against women physicians. ${ }^{17}$ Recently, various solutions have been proposed to overcome these biases. In this review, we discuss how social media may serve as tools for advancing WIC and foster gender equality and diversity.

\section{The Rise of Social Media and Hashtag Activism}

The use of social media in the field of academic medicine has increased over the past few years, ${ }^{19}$ with the creation of online communities of physicians in the field of medicine and its specialties. The rise of social media has influenced how female physicians communicate. ${ }^{20}$ Virtual platforms are important avenues where female physicians have united to create greater female representation in regard to gender issues and advocacy efforts. An important example of these efforts is the hashtag activism used to bridge the gap in gender issues (Fig. 2). Initially started by the non-medical community, social media hashtags such as \#metoo, which trended in 85 countries, ${ }^{21}$ allowed for the amplification of women's voices, owing to the hashtags' global accessibility and ease of use. Women in the field of medicine (WIM) participated, using the hashtag \#metoomedicine. Within cardiology, the hashtags \#WIC, \#ACCWIC (American College of Cardiology Women in Cardiology), and \#AHAWIC (American Heart Association Women in Cardiology) have been used to collectively represent the voices of WIC. The use of "hashtag activism" has allowed WIC to connect through a common experience, but more importantly, to share that experience on a public platform through Twitter, elevating their voice and creating allies in the process. This social media activism has been used to promote a culture shift within the field of cardiology, creating a more inclusive, respectful work environment. ${ }^{22}$ 


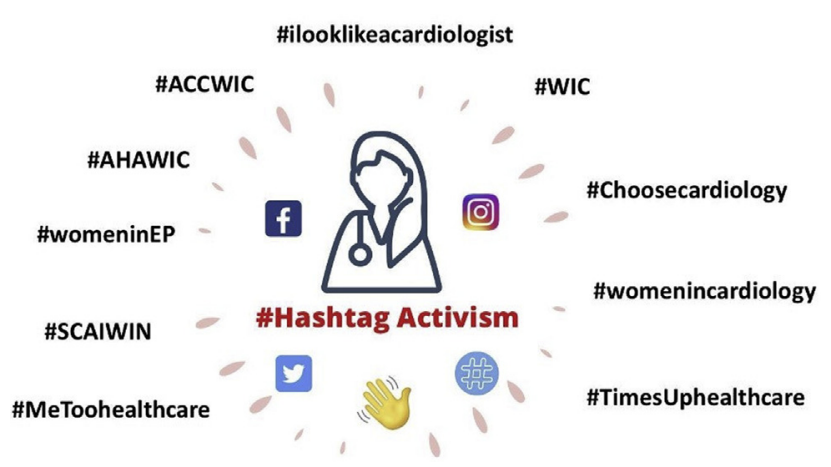

Figure 2. The phrases of "hashtag activism"19-21: a representation of the frequently used hashtags within the women in cardiology (WIC) network. ACCWIC, American College of Cardiology Women in Cardiology; AHAWIC, American Heart Association Women in Cardiology; SCAIWIN, Society for Cardiovascular Angiography and Interventions Women in Innovations.

Social media provide a unique means of fostering collaboration, networking, mentorship, and dissemination of research, which may help to both increase representation and close the gender gap within the field. ${ }^{19,23}$ It has been shown that platforms such as Twitter greatly extend the reach of tweets and journal articles related to issues of gender equity and increase the impact of publications and the number of paper downloads. ${ }^{23,24}$ Thus, social media serve to further amplify gender-equality activism by facilitating the conversations surrounding gender equity and proposing solutions to self-identified issues by the virtual community of female physicians and their allies. ${ }^{19}$

As the presence of WIC on social media increases, along with the use of hashtags, it is imperative to understand how these increases are impacting discussions. Recent analysis of hashtags, such as \#WIC and \#WomeninMedicine, has shown an upward trend in usage in the cardiology Twitter community. ${ }^{24}$ Analysis of 6 key hashtag phrases identified showed that, over a period of 3 years (2016-2019), there was a $706 \%$ increase in tweets and retweets of tagged posts, a $207 \%$ increase in impressions, and a $440 \%$ global increase in Twitter users sending tweets within the "Women in Cardiology Twitter Network." ${ }^{22}$ The momentous increase in tweet shares and global impressions signifies the power of hashtags to reach a target audience. The nature of what is shared on the virtual platform has also shifted over the years, with WIC speaking about the real-life stories of gender discrimination such as the pay gap in comparison to what their male colleagues in cardiology earn, lack of respect by patients, staff, and colleagues, skepticism from patients about their qualifications simply because they are women, being recognized as a "girl" and not as a doctor, lack of consideration and/or accommodation during and after pregnancies, and other issues (Fig. 3) ${ }^{24}$ By using gender-activist hashtags in combination with specialtyfocused hashtags, WIC can put their experiences of gender inequity and sexual harassment front-and-center on the pages of their cardiology peers who need to confront, evaluate, and adjust their behaviour and hopefully impact the culture they are a part of at their institution. An analysis of various womencentric hashtags, such as \#WIC, revealed that communitybuilding was the focus of most posts by social media users using the hashtag. ${ }^{22}$ In the percentage of both original tweets and "amplified" tweets (via re-tweets), professional development and mentorship were key content focuses of WIC and those within the cardiology network. ${ }^{22}$ Social media have therefore increased the visibility of WIC achievements and increased awareness of gender discrimination within the workplace, and generated solidarity. ${ }^{22}$

\section{WIC Networks on Social Media}

In conjunction with Twitter, which promotes social movements through the grouping of topics by use of "hashtags,"25,26 other platforms, such as Facebook, have become

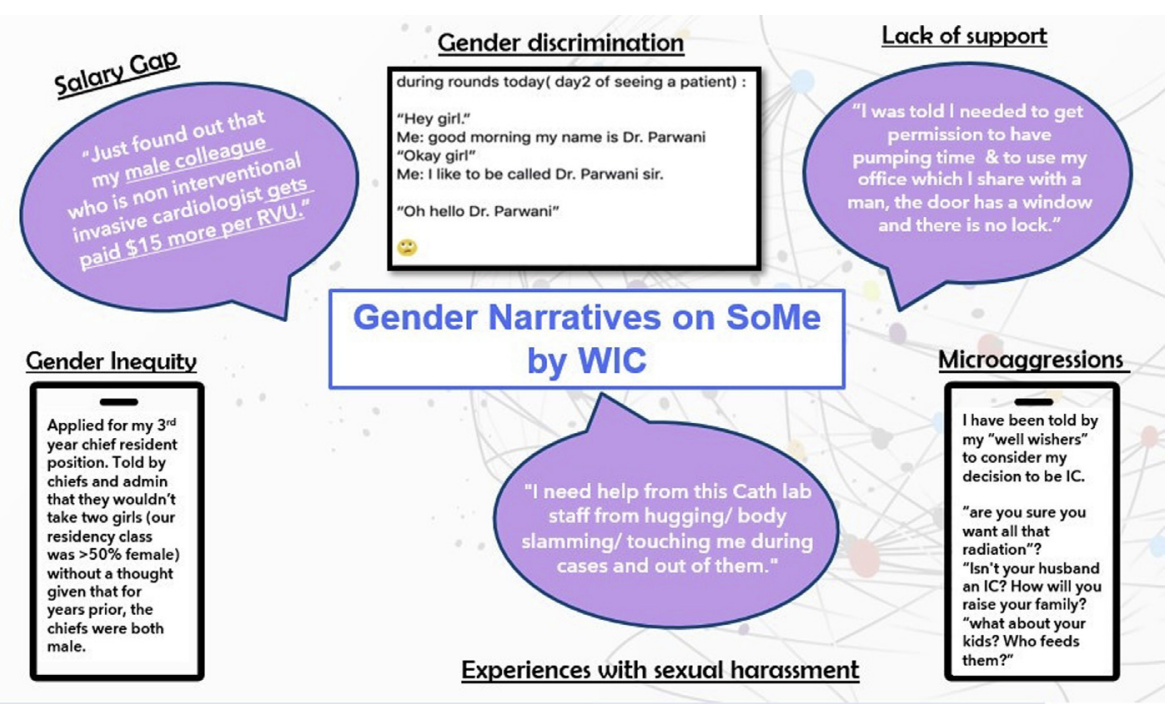

Figure 3. Visual representation of gender narratives on social media by women in cardiology. ${ }^{26,38,39}$ SoMe, social media; WIC, women in cardiology. 
home to growing communities in which WIC can communicate and support one another. Notably, Facebook's WIC group has become a hub for women to communicate freely in an open forum outside the workplace and to connect with WIC beyond their immediate workplace. ${ }^{27}$ The private Facebook group has allowed WIC to have candid discussions and exchange shared experiences without the fear of judgment from male or unfriendly onlookers. In this closed group, WIC can further seek support regarding the difficulties they face, such as managing the "second shift" or navigating workplace discrimination. ${ }^{27,28}$

Emerging social media platforms such as Clubhouse ${ }^{29}$ further expand the connections women make to one another and broaden the reach of gender-equality activism, becoming popular among healthcare professionals and being used as an educational tool. Any user on the platform can form a "club," which is generally focused on a more specific topic to appeal to those who are interested. For example, the WIM and WIC clubs have gained over 1000 and 440 members, respectively, in the past 3 months, facilitating conversations such as "sexism in healthcare," "the leaky pipeline for women in medicine," and other topics surrounding the disparities that women in healthcare face and how to combat them. ${ }^{19}$ These community spaces foster a sense of solidarity among female health professionals with similar experiences of gender bias and allow for open and extemporaneous communication regarding positive strides that have been made; having the ability to speak up and share experiences and particularly to have men in the audience listen to the conversation unfiltered and participate, may help stimulate further change in the misogynistic culture that has persisted in medicine and cardiology.

\section{The Impact of Gendered Conversations on Social Media}

It is well documented that social media greatly influences perceptions of self-worth and self-concept of the members of younger generations, who consume social media constantly throughout their days. ${ }^{29}$ Moreover, social media are particularly influential for young women, impacting their preferences, social behaviours, and even academic interests as early as elementary school. ${ }^{30}$ Representation of genders in media not only influences not only gender roles on a societal scale but also how women perceive themselves. This process becomes a cycle in which girls do not see themselves represented in medical fields, and therefor self-select out of these professions by tailoring-either consciously or subconsciously - their expressed interests to match those in fields that are traditionally female-dominated. ${ }^{31}$ The solution to this vicious cycle is to promote and publicize WIM and WIC. Social media, such as television and film, influence cultural norms by modeling gender-appropriate behavior. ${ }^{30}$ Social media's ease of accessibility and rapid turnover time, including its accessibility to the broader public outside of medicine, makes it a remarkable conduit of social influence. $^{29}$ In $2018,93.5 \%$ of medical students reported using some form of social media, including Facebook, Twitter, and LinkedIn. ${ }^{32}$ These numbers continue to rise as novel social media platforms develop and social media use becomes more pervasive, particularly with the shift to online classes and work-from-home that has occurred during the coronavirus disease 2019 (COVID-19) pandemic. ${ }^{33}$ The use of electronic media for work in the form of telemedicine in 2020 was paralleled by an increase in use of social media for personal engagement as well, thereby amplifying the presence of both aspiring physicians and current physicians on social media platforms. ${ }^{33}$ This increased presence on social media provides an opportunity to further spotlight WIC and amplify their presence. As WIC, as well as women from other subspecialties of medicine, continue to grow their public presence on social media, young women who frequently use social media sites such as Twitter, Facebook, Instagram, and now Clubhouse, will hear the amplification of voices and perhaps will visualize themselves represented in medicine in the future. This growth will help minimize self-selection of women out of male-dominated subspecialties such as cardiology; hopefully, through representation and positive role modeling, this growth will help eliminate the underrepresentation of WIC.

Although these social media platforms provide the ability for women to talk freely about their experiences and bring light to the issues they face, they also encourage being allies with men, with the use of hashtags such as \#HeforShe, which allows male colleagues to be part of the movement to change the misogynistic culture. With the emergence of social media platforms such as Clubhouse, men can express their support in real time, actively participate in conversations, and listen to the problems women in medicine face professionally and personally. Hashtag activism has also allowed for identification and public criticism of all-male panels (also known as a "manel") in conferences and meetings. ${ }^{34}$ The importance of having gender diversity at medical conferences has been continuously highlighted on social media, with a slow increase in women in key speaking roles, but much work remains to be done. ${ }^{35}$ With social media, medical professionals can help garner a community that helps bring light to issues such as these and ensure gender diversity in all facets of medicine. ${ }^{36}$

Online tweeting about sexism has also been shown to promote the well-being of the activists via promotion of positive properties of gender identity. ${ }^{37}$ While sharing their stories on social media, women take pride in identifying themselves as women while interacting with other women and thriving in that environment, one in which women can be heard and share their experiences and not feel alone. ${ }^{38}$ Indeed, documenting the pervasiveness of these negative experiences is the first step in effecting change.

\section{Conclusion}

For a long time, WIC issues have been in the blind spot of the cardiovascular community. Social media is that highquality mirror that makes the \#WIC blind spot visible. The power of social media to influence societal norms and cultural beliefs, and to eliminate rigid gender roles, is immense; this fact, coupled with the ever-changing nature of social media and its ability to leverage current social change trends, presents an opportunity for culture change for WIC. By using social media to highlight their presence and amplify their voices, WIC perhaps will be able to shift the social perceptions of cardiology as a male-dominated space to one where women can coexist and even thrive. This process will help recruit more women into the field of cardiology, create allies in men, promote changes to the 
gender-biased culture in the workplace, and hopefully seal the leaky pipeline (Central Illustration).

\section{Funding Sources}

The authors have no funding sources to declare.

\section{Disclosures}

Dr Parwani serves as a Guest Editor to Journal of the American College of Cardiology (JACC): Case Reports. Dr Choi serves as Social Media Editor for JACC: Cardiovascular Imaging. Dr Grapsa serves as an Editor-in-Chief for JACC: Case Reports. Dr Michos serves as co-Editor-in-Chief for the American Journal of Preventive Cardiology. All the other authors have no conflicts of interest to disclose.

\section{References}

1. Association of American Medical Colleges. The majority of U.S. medical students are women, new data show. Available at: https://www.aamc.org/ news-insights/press-releases/majority-us-medical-students-are-women-newdata-show. Accessed May 27, 2021.

2. Rossi AM, Pilote L. Let's talk about sex....and gender! Circ Cardiovasc Qual Outcomes 2016;9(2 suppl 1):S100-1.

3. Kaye EC. Misogyny in medicine. N Engl J Med 2021;384:2267-9.

4. Benya FF, Widnall SE, Johnson PA, et al., eds. Sexual Harassment of Women: Climate, Culture, and Consequences in Academic Sciences, Engineering, and Medicine. Washington, DC: National Academies Press, 2018.

5. Sanghavi M. Women in cardiology: introspection into the under-representation. Circ Cardiovasc Qual Outcomes 2014;7:188-90.

6. Mansour C, Tamirisa KP, Lundberg G, et al. Sexual harassment, victim blaming, and the potential impact on women in cardiology. JACC Case Rep 2021;3:978-81.

7. Association of American Medical Colleges. Physician specialty data report. Available at: https://www.aamc.org/data-reports/workforce/ interactive-data/active-physicians-sex-and-specialty-2019. Accessed May 27, 2021.

8. Accreditation Council for Graduate Medical Education. ACGME data resource book. Available at: https://www.acgme.org/about-us/publications -and-resources/graduate-medical-education-data-resource-book/. Accessed June 6, 2021.

9. Blumenthal DM, Olenski AR, Yeh RW, et al. Sex differences in faculty rank among academic cardiologists in the United States. Circulation 2017;135:506-17.

10. Balasubramanian S, Saberi S, Yu S, et al. Women representation among cardiology journal editorial boards. Circulation 2020;141:603-5.

11. Jena $A B$, Olenski AR, Blumenthal DM. Sex differences in physician salary in US public medical schools. JAMA Intern Med 2016;176: 1294-304.

12. Shah RU. The $\$ 2.5$ million wage gap in cardiology. JAMA Cardiol 2018;3:674-6.

13. Glynn SJ. An unequal division of labor: how equitable workplace policies would benefit working mothers. Available at: https://www.american progress.org/issues/women/reports/2018/05/18/450972/unequal-divisionlabor/. Accessed June 6, 2021.
14. Ganguli I, Sheridan B, Gray J, et al. Physician work hours and the gender pay gap—evidence from primary care. N Engl J Med 2020;383:1349-57.

15. El Arnaout N, Chehab RF, Rafii B, Alameddine M. Gender equity in planning, development and management of human resources for health: a scoping review. Hum Resour Health 2019;17:52.

16. Lyu G, Davids JS, Scully RE, Melnitchouk N. Association of domestic responsibilities with career satisfaction for physician mothers in procedural vs nonprocedural fields. JAMA Surg 2019;154:689-95.

17. Sharma G, Douglas PS, Hayes SN, et al. Global prevalence and impact of hostility, discrimination, and harassment in the cardiology workplace. J Am Coll Cardiol 2021;77:2398-409.

18. Silver JK, Rowe M, Sinha MS, et al. Micro-inequities in medicine. PM R 2018;10:1106-14.

19. Parwani P, Choi AD, Lopez-Mattei J, et al. Understanding social media: opportunities for cardiovascular medicine. J Am Coll Cardiol 2019;73: 1089-93.

20. Cawcutt KA, Erdahl LM, Englander MJ, et al. Use of a coordinated social media strategy to improve dissemination of research and collect solutions related to workforce gender equity. J Women's Health 2019;28: $849-62$.

21. Jewett C. Women in medicine shout \#MeToo about sexual harassment at work. Washington Post.com. March 18, 2018. Available at: https://www.washingtonpost.com/national/health-science/women-inmedicine-shout-metoo-about-sexual-harassment-at-work/2018/03/16/ 81673f82-0c37-11e8-8b0d-891602206fb7_story.html?noredirect = on\&utm_term $=.4$ edc30d2a3a9. Accessed June 13, 2021.

22. Chandra NV, Hsiao R, Shapiro H, et al. Women in cardiology Twitter network: an analysis of a global professional virtual community from 2016 to 2019. J Am Heart Assoc 2021;10. e019321.

23. Parwani P, Martin GP, Mohamed MO, et al. Relationship of altmetric attention score to overall citations and downloads for papers published in JACC. J Am Coll Cardiol 2020;76:757-9.

24. Parwani P, Faza NN, Fudim M, et al. JACC: Case Reports: reflections from 1 year on social media. JACC Case Rep 2020;2:1818-23.

25. Kuehn BM. Social media becomes a growing force in cardiology. Circulation 2020;140:790-2.

26. Yeung D. Social media as a catalyst for policy action and social change for health and well-being: viewpoint. J Med Internet Res 2018;20:e94.

27. Facebook. Women in cardiology. Available at: https://www.facebook. com/groups/745868888796666/. Accessed June 1, 2021.

28. Parwani P, Han JK, Singh T, Volgman AS, Grapsa J. Raft of otters: women in cardiology: let us stick together. JACC Case Rep 2020;2: 2040-3.

29. Alpha Exploration Co. Clubhouse mobile application, version 0.1.39. Available at: https://www.joinclubhouse.com/press. Accessed June 1, 2021.

30. Bhatt M, Blakley J, Mohanty N, Payne R. How media shapes perceptions of girls and women. Available at: https://s3.amazonaws.com/ feminc-website/Reports/femWhitePaperHighResFinal.pdf. Accessed June 1, 2021.

31. Olsson M, Martiny SE. Does exposure to counterstereotypical role models influence girls' and women's gender stereotypes and career choices? A review of social psychological research. Front Psychol 2018;9: 2264. 
32. Karim F, Oyewande A, Abdalla LF, Chaudhry Ehsanullah R, Khan S. Social media use and its connection to mental health: a systematic review. Cureus 2020;15:e8627.

33. Shillcutt SK, Silver JK. Social media and advancement of women physicians. N Engl J Med 2018;378:2342-5.

34. Katz M, Nandi N. Social media and medical education in the context of the COVID-19 pandemic: scoping review. JMIR Med Educ 2021;7:e25892.

35. Kibbe MR, Kapadia MR. Underrepresentation of women at academic medical conferences- "manels" must stop. JAMA Netw Open 2020;3: e2018676.
36. Corsini EM, Boeck M, Hughes KA, et al. Global impact of social media on women in surgery. Am Surg 2020;86:152-7.

37. Foster MD. Tweeting about sexism: the well-being benefits of a social media collective action. Br J Soc Psychol 2015;54:629-47.

38. Foster MD. "Use it or lose it": how online activism moderates the protective properties of gender identity for well-being. Comput Human Behav 2019;96:163-73.

39. Twitter. Women in cardiology. Available at: https://twitter.com/ womencardiology?lang = en. Accessed June 1, 2021. 